\title{
Rheology of the Tectonosphere \\ as Inferred from Seismic Aftershock Sequences
}

\author{
G. Ranalli $(*)$ - A. E. ScheidegGer $(* *)$
}

Ricevuto il 9 Novembre 1969

\begin{abstract}
SUMmary. - Some rheological models of the mechanism of aftershock occurrence, namely, Benioff's, Pshennikov's, and Mogi's models, are examined in the light of the established laws governing the phenomenology of the process. It is concluded that none of them explains fully the aftershock mechanism. Thus, a new creep model is proposed, according to which aftershocks are the discontinuous manifestation of the overall plastic creep by which the rock readjusts itself to the stress distribution left by the main shock. The creep model affords a new large-scale picture of the rheology of the tectonosphere.
\end{abstract}

Riassunto. - Alcuni modelli reologici del meccanismo delle repliche di terremoti (modelli di Benioff, di Pshennikov e di Mogi) sono esaminati alla luce delle leggi che regolano la fenomenologia del processo. Nessun modello spiega completamente il meccanismo in azione. Quindi si propone un nuovo modello ("creep model"), secondo cui le repliche sono la manifestazione discontinua della deformazione plastica cui la roccia è soggetta a causa della distribuzione delle tensioni lasciate dalla scossa principale. Questo modello fornisce una nuova visione globale della reologia della tettonosfera.

\section{INTRODUCTION.}

The problem of the rheological response of the tectonically important layers of the Earth (crust and upper mantle) to the action of

(*) Now at the Atlantic Oceanographic Laboratory, Bedford Institute, Dartmouth, Nova Scotia, Canada.

$\left({ }^{* *}\right)$ Department of Mining, Metallurgy and Petroleum Engineering University of Illinois at Urbana-Champaign-Urbana, Illinois. 
tectonic stresses is an extremely important one; it is, in fact, the basic problem of geodynamics. Once the rheological response-conditions are known, it is, in principle, possible to explain the mechanics of mountainbuilding.

If the rheological conditions obtaining in the Earth are to be studied, one has to distinguish between various time ranges, inasmuch as the response of any material to quickly and slowly changing stresses need not be the same. With regard to the Earth, it has turned out to be convenient to distinguish three time ranges: "Short" (up to four hours), "intermediate" (four hours to 15,000 years), and "long" (longer than 15,000 years). One of us (1) has summarized what was known about the rheology of the Earth about twelve years ago. For the short time range, the information was basically obtained from the propagation of seismic waves, for the intermediate time range from the earthquake aftershock sequences, and for the long time range from the long-term isostatic adjustment of once glaciated areas.

Meanwhile, much new information has come to light on the phenomenology of aftershock sequences of earthquakes. This information has an important bearing upon the rheological conditions in the crust and upper mantle of the Earth in the "intermediate" time range. Thus, it is the purpose of the present paper to re-analyze the rheology of the Earth in the intermediate time range in the light of the new phenomenological information on aftershock sequences. It will be found that it turns out that the Earth in this time range can no longer be regarded as a Kelvin body with elastic afterworking, but that it must be regarded as a " macroscopically" plastic body which adjusts itself to a redistribution of stresses by logarithmic creep.

\section{Statistical LAWS GOVERNING THE PHENOMENOLOGY OF AFTERShock SEQUENCES.}

The observed features of aftershock sequences obviously depend upon the rheological properties of the material in the focal region. Any theory of the aftershock mechanism must account for these observations. The laws describing the phenomenology of aftershock occurrence are the following.

(1) Time-frequency law (Omori's law) (2). 
The frequency $n$ of aftershocks per unit time decreases hyperbolically after the origin time of the main shock,

$$
n(t)=a^{t-\beta}
$$

where $\alpha$ and $\beta$ are constants, and $t$ is in days. Usually $\beta$ is equal to or slightly greater than unity. The law is valid for $t \leqslant 100$.

(2) Magnitude stability in time $\left({ }^{3}\right)$. The mean magnitude $M^{\prime}$ of groups of 10 successive aftershocks within the same sequence shows only random fluctuations about the overall mean magnitude $\bar{M}$ of the whole sequence.

(3) Magnitude-frequency distribution ( $\left.{ }^{4}\right)$. The magnitude-frequency distribution is of the form

$$
N(M)=K e^{-b^{\prime}(M-M *)}
$$

where $N(\boldsymbol{M})$ is the number of aftershocks with magnitude greater than or equal to $M$, and $M^{*}$ is the minimum detected magnitude in the sequence. Usually the coefficient $b^{\prime}$ is expressed as

$$
b=b^{\prime} \log e
$$

and $b$ is generally slightly less than unity.

To these laws it must be added that aftershocks are predominantly crustal phenomena, although deep sequences also occur.

The above laws, as is indicated by the cited references, were proposed on several occasions in the literature. An accurate statistical analysis was made by Ranalli (5) on 15 aftershock sequences which confirmed these laws.

As noted, the observed laws must stand at the basis of any interpretation of data in terms of the rheology of the upper mantle and crust of the Earth. The possible models for such an interpretation will be discussed next.

BENIOFF'S MODEL.

An explanation of the mechanism of aftershock occurrence based upon an analogy with continuous rock deformation has been proposed by Benioff ( $\left.{ }^{6}\right)$. The fundamental idea is that aftershocks are caused by elastic afterworking of the Kelvin type and are the discontinuous equivalent of continuous strain recovery. The rocks in the focal 
volume are considered homogeneous, apart from the presence of a fault. The aftershock-generating mechanism may be illustrated by means of the rheological model shown in Fig. 1. The strength element $s$ represents the fault; $\mu_{1}$ the elastic element; $\left(\mu_{2}, \eta_{2}\right)$ the firmoviscous element; $\eta_{\mathrm{L}}$ a secular high-viscosity resistive element which is assumed not to move appreciably during the sequence.

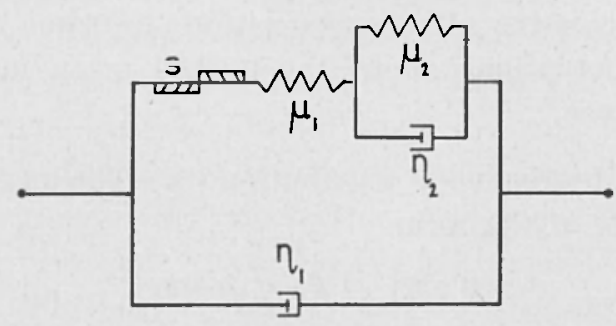

Fig. 1 - Benioff's model of the aftershock mechanism.

If the model is slowly compressed by an applied tectonic force, at a certain state the static friction between the fault walls is overcome and sliding occurs (main shock); the elastic element $\mu_{1}$ is therefore discharged. This element, however, is again stressed by the slow recovery of the firmoviscous element $\left(\mu_{2}, \eta_{2}\right)$; consequently, the stresses are again built up at the fault, and sliding occurs again when they overcome the friction (first aftershock). The process is repeated until the residual strain in the Kelvin element is fully relieved, or, more precisely, when the stress in $\mu_{1}$ becomes too low to overcome the friction at the fault.

While Benioff's model explains the occurrence of aftershocks following a large earthquake, it does not explain all the observed features of an aftershock sequence. The preexistence of a fault is not a necessary condition for the occurrence of seismic shocks. The model can be easily modified by assuming that the element $s$ is simply the strength of the focal rock (or the strength along a plane of weakness in the unfractured rock); then the fault would be created by the main shock. Nevertheless, all aftershocks would still occur along the same fault. This is certainly true in some sequences, but not in others.

Benioff ( ${ }^{6}$ ) constructed "strain release curves". Let $V_{0}$ be the earthquake (or focal) volume, i.e., the volume of rocks where energy is stored before an earthquake and through which it is discharged. 
Assuming that the strain components have an average constant value throughout $V_{o}$, considering the material in $V_{o}$ to be elastic, homogeneous and isotropic, and under suitable thermodynamic conditions, the total compressional and distortional strain energies in $V_{0}$ are respectively

$$
\begin{aligned}
& W_{c}=\frac{1}{2} k \theta^{2} V_{\circ} \\
& W_{d}=\mu \varepsilon^{o{ }^{2} i j} V_{0}=\mu\left(\varepsilon_{i j}{ }^{2}-\frac{1}{3} \theta^{2}\right) V_{\circ}
\end{aligned}
$$

where $\mu$ is the rigidity, $k$ is the bulk modulus, $\varepsilon$ is the strain tensor, $\varepsilon^{\circ}$ is the strain-deviator, and $\theta=\varepsilon_{i t}$ is the cubical dilatation. While only the distortional energy contributes to fracture, both types are released as seismic energy $J$, that is,

$$
J=q\left(W_{c}+W_{d}\right)=q W
$$

where $q$ is the seismic loss ratio, accounting for that part of the strain energy which is dissipated by mechanical friction and the like as heat. Form [3] and [4] one has

$$
J=\frac{1}{2} k q \theta^{2} V_{o}+\mu q\left(\varepsilon^{2} i j-\frac{1}{3} \theta^{2}\right) V_{o}
$$

and, since in the Earth's crust $k \simeq \frac{5}{\mathbf{J}} \mu\left({ }^{7}\right)$ :

$$
J=\mu q\left(\varepsilon^{2} i j+\frac{1}{2} \theta^{2}\right) V_{o}
$$

which, in order to comply with Benioff's original notation, may be written as follows

$$
J=\frac{1}{2} \mu q \varepsilon^{-2} V_{o}
$$

where

$$
\varepsilon^{-2}=2 \varepsilon^{2} i j+\theta^{2} .
$$

Taking square roots in equation [5] one obtains for the strain

$$
\bar{\varepsilon}=\left(\frac{2 J}{\mu q V_{0}}\right)^{1 / 2}
$$


Benioff ( $\left.{ }^{(}\right)$assumed that, in an aftershock sequence, $q$ does not change from shock to shock, and his model implies that the energy is always discharged through the same volume $V_{o}$. Consequently the factor

$$
C=\left(\frac{2}{\mu q V_{o}}\right)^{1 / 2}
$$

appearing in equation [6] is constant and as a result strain is proportional to the square root of the released seismic energy. Therefore, if $\bar{\varepsilon}_{i}=C J_{i}^{1 / 2}$ is the strain released by the $i$-th aftershock, the cumulative strain release after the $n$-th shock is

$$
S_{n}=\sum_{i=1}^{n} \bar{\varepsilon}_{i}=C \sum_{i=1}^{n} J_{i}^{1 / 2}
$$

A strain release curve can then be constructed by fitting a smooth curve $S(t)$ to the step function $S_{n}$. Benioff $\left({ }^{6}\right)$ applied this procedure to aftershock sequences and to series of independent earthquakes. In aftershock sequences, however, because of the time stability of the mean magnitude $\left({ }^{3,5}\right)$ the expected value of any function of magnitude (such as the energy $J)$ is constant in time, $E(J)=J(\bar{M})$, apart from individual fluctuations which can be quite large but are completely random and therefore are of no interest when considering the overall trend of the curve. This implies that, on the average, each aftershock in the sequence contributes the same amount to the cumulative strain release $S_{n}$. It is assumed that undetected aftershocks do not affect the shape of the curve. The smooth curve $S(t)$ is given by

$$
S(t)=\int_{0}^{t} n(t)[J(M)]^{1 / 2} d t=[J(\bar{M})]^{1 / 2} \int_{0}^{t} n(t) d t=A \int_{0}^{t} n(t) d t
$$

where $A$ is a constant. Therefore the cumulative frequency of aftershocks is equivalent to the cumulative strain release.

If Benioff's model were correct, the cumulative strain release (or the cumulative frequency of aftershocks) should be exponential due to the presence of the Kelvin element. However, the cumulative frequency is adequately represented by a quasi-logarithmic curve, at least in the first part of a sequence. By differentiation, it can be seen 
that Benioff's model predicts an exponential decay of the aftershock frequency:

$$
n(t)=\alpha^{\prime} e^{-\beta^{\prime} t} .
$$

It turns out that the best-fitting exponential curve [9] fits the data much more poorly that the best-fitting hyperbolic curve [1]. As an example, Fig. 2 shows the decay of $n(t)$ for the Alaska, 196t, after-

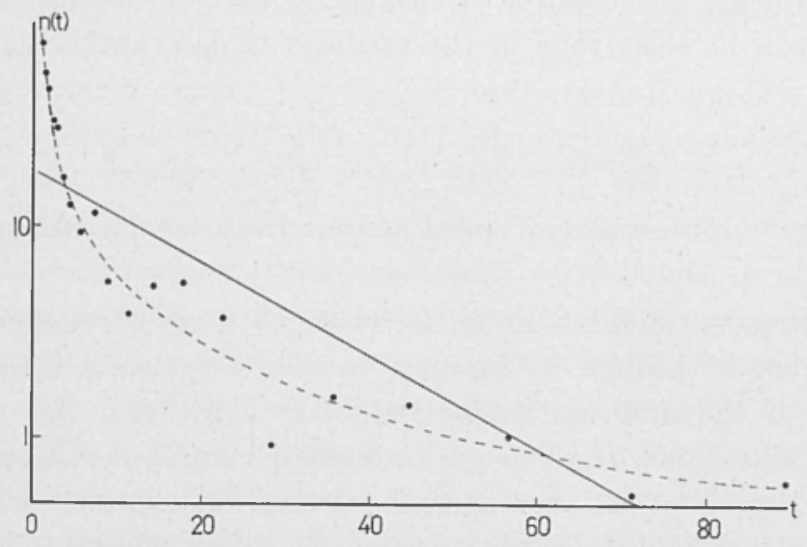

Fig. 2 - Comparison between hyperbolic and exponential decay of aftershock frequency.

shock sequence $\left(^{5}\right)$. The solid line represents the fitted exponential curve, the dotted line the fitted hyperbolic curve. It is clear that the second curve gives a better approximation to the trend of the data points" (full circles). Consequently, if the postulated equivalence between continuous and discontinuous strain release is valid, these results indicate that a model based upon elastic afterworking of the Kelvin type does not account for the observed facts.

\section{PshenNikov's MODEL.}

Pshennikov ( $\left.{ }^{8}\right)$ has tried to explain the aftershock mechanism in terms of the properties of a Maxwell-body. As in Benioff's model, it is assumed that the main shock releases only part of the energy which has been accumulated in time within the focal volume. The residual energy is partly released in the form of aftershocks and partly dissipated as heat or in continuous creep during an extended period of time. 
Pshennikov concludes that the curve of released stress is well represented by the stress relaxation curve of a Maxwell-body (that is, by an exponential curve) with time-dependent relaxation time.

The details of Pshennikov's method of constructing the stress release curve are not of much significance in the present context. The main objection to the model, as Kuznetsova and Popov ( ${ }^{9}$ ) have emphasized, is that the very presence of aftershocks in a Maxwell body is difficult to explain. Considering the rheological model in Fig. 3, it can be seen that, if the medium is deformed at a constant

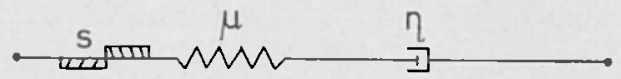

Fig. 3 - Pshennikov's model of the aftershock mechanism.

rate, the stresses increase until the strength limit is reached and are then unloaded through $s$, thereby causing a seismic shock. Subsequently, if the deformation of the body continues, the process is repeated. But since the viscous element $\eta$ cannot move appreciably in a short time interval, such as that existing between two consecutive aftershocks, a second shock may occur only if new energy is introduced into the system by the externally applied tectonic force. The latter, by all accounts, builds up far too slowly to be able to increase again the stresses beyond the strength limit in a few hours or less. This is true also if it is assumed that the strength at the fault is somewhat decreased after the first shock. In other words, an aftershock sequence is (geologically speaking) so short that it is not possible to envisage a considerable energy build-up at the expense of some outside source occurring during the length of the sequence. Furthermore, as shown in the preceding section, an exponential decay curve does not fit the data as well as a hyperbolic curve.

Pshennikov's model, therefore, could possible depict the occurrence of series of independent seismic events occurring at approximately the same spot, but is inadequate to account for the mechanism of aftershock occurrence.

MOGI'S MODEL.

Mogi (10-14) has studied experimentally the fracturing of rocks and has reached some conclusions about the nature of the earthquake 
process based upon an extrapolation of results obtained in the laboratory to the conditions pertaining in the Earth's crust. The basic assumption underlying his work is that it is precisely the heterogeneity of the crust which plays a role in aftershock occurrence, while in the two models discussed above the rock in the focal volume was considered essentially homogeneous, apart from the presence of a fault. Moreover, the macroscopic heterogeneities of the crust are assumed to be equivalent to the small-scale heterogeneities of rocks experimented upon in the laboratory.

Using various types of granite, andesite and pumice as models of a heterogeneous crust, and analyzing the elastic shocks caused by their microfracturing under stress, Mogi (11) observed a suggestive correspondence between creep and microfracturing. The first stage of continuous deformation (transient creep) corresponds to a decreasing frequency of microfractures which occur immediately after the application of stress; in the second stage (steady-state creep) no microfractures are observed; the third stage of accelerating creep leading to macroscopic fracture corresponds to a rapidly increasing frequency of microfractures. The conclusion that rock creep is related to timedependent microfracturing is supported also by other experimental eviderıce $\left({ }^{15}\right.$ 17). Thus, a correspondence between continuous and discontinuous rock deformation has been established. If the extrapolation to large-scale conditions in the crust is correct, it lends a new plausibility to the attempt of correlating aseismic and seismic phenomena.

It is also interesting to note that the magnitude-frequency distribution of the microfractures in creep in heterogeneous rocks satisfies the same magnitude-frequency law followed by both independent earthquakes and aftershock sequences. This fact again points to the fundamental character of the partition of energy among elastic shocks.

Small-scale fracturing of rock in the laboratory has been observed by Mogi (12-14) also after the occurrence of the main fracture. In his experiments, a rock specimen is stressed by means of a stress source located therein at a small depth below the free surface. The pattern of activity varies according to the structure of the medium; however, apart from the case of an extremely heterogeneous material (which shows a swarm of elastic shocks with no clearly defined main shock), the main fracture is always followed by a sequence of minor fractures whose frequency decreases gradually in time. The fractured region is always located between the stress source and the free surface. If 
the stress source is too deeply buried, no (or very few) minor fractures follow the main shock. The presence of a free surface, therefore, seems to influence the occurrence of secondary fractures. This would account for the predominantly shallow character of aftershock sequences.

If essentially similar processes occur in the Earth's crust, the occurrence of aftershocks can be explained as follows. When, under continuously accumulating tectonic stresses the strength of the focal rock is reached, fracture occurs and a major part of the strain energy is liberated as seismic energy (main shock). At the same time, new planes of weakness and incipient fractures appear at irregular points in the focal volume. Consequently, as Mogi (10) states verbatim:

"Although the mean stress in the region will decrease remarkably with the occurrence of the main shock, the concentrated stress at these irregular points will increase suddenly immediately after the main shock. Thus, many local fractures will occur at these points following the main shock".

It is to be noted that the idea that aftershocks are caused solely by the redistribution of stresses inside the medium after the main shock is by no means new. Jeffreys $\left({ }^{18}\right)$ reached the same conclusion in the study of the aftershocks of the 1927 Tango (Japan) earthquake. Mogi's contribution to the problem consists mainly of the experimental evidence brought forth.

Microfractures occurring after the main fracture of rock in compression have been observed experimentally also by Scholz (19). If the sample is isolated after fracture, microfracturing activity decreases hyperbolically in time, as in earthquake aftershock sequences. Scholz's idea - similar to Mogi's - is that such microfractures occur at points of stress concentration in the heterogeneous medium. The strength of the rock is assumed to be time-dependent, and the stress at any point is regarded as composed of a constant part (the mean or applied stress) plus a random part due to local conditions. It is reasonable to assume that time-dependent microfracturing is also associated with the deformation of rock in situ. Aftershocks would then be the largescale equivalents of experimental microfractures; in other words, microfracturing is a scale model of seismicity.

The statistical similarities between creep and microfracturing in laboratory specimens and seismic processes are indeed striking. In Mogi's model, however, most (if not all) aftershocks would be caused by the occurrence of new fractures. Thus, the model does not account for 
the sequences in which activity along the same fault is predominant; in general, the role of friction and of stickslip phenomena is neglected. Furthermore, the rheological characteristics of the rock in the focal volume do not affect the model, as long as the medium is capable of fracture followed by a stress drop. Thus, the only conclusion based on Mogi's model that can be reached about the rheological properties of the material in the focal volume is that it must have a strength limit and be capable of (brittle) fracture.

\section{CONCLUSTON: A NEW CREEP MODEL.}

None of the models discussed above gives a complete picture of the aftershock process. Mogi's model, however, fits best the observed features of aftershock sequences inasmuch as it does not contradict any of the established phenomenological laws. On the other hand, no definite statement about the rheological behavior of the material in the earthquake volume can be based upon it. What we are referring to is the rheology of the material in the earthquake volume $V_{0}$ considered as a whole; that is to say, we identify $V_{0}$ with the total aftershock volume, regard it as a rheological unity, and consider its (average) rheological properties.

According to Benioff $\left({ }^{6}\right)$, the material in $V_{o}$ is essentially elastic and therefore strain is proportional to the square root of the energy, cf. formula [6]. Mogi (14) assumes only that such a material is capable of fracture, which leaves unanswered the question of its overall rheological behavior. In the present context, some results of Bath and Duda $\left(^{20}\right)$ are very important. In a study of several aftershock sequences which occurred in different geographical regions, these authors have concluded on the basis of empirical evidence that the volume affected by a shock increases with magnitude according to the relation $\left(V\right.$ in $\left.\mathrm{cm}^{3}\right)$

$$
\log V=9.6+1.5 M
$$

where $M$ is the surface wave magnitude. Using this fact and the common magnitude-energy relation

$$
\log J=11.8+1.5 M
$$

it is readily seen from equation [6] that strain release is independent of magnitude. (We assume that $\mu$ and $q$ are at least approximately 
constant). It must be noted that the idea that the energy released by a seimic shock depends mainly on the rock volume affected and not on the strain had already been put forward by Tsuboi (21).

Even if the volume-magnitude relation [9] fails to hold exactly, it seems clear that large shocks affect a larger rock volume than small ones. It is reasonable to assume that a rock should break when it is strained up to a certain level, and if magnitude is strain-dependent, it is difficult to see why the same rock should be subject to shocks of different magnitudes. Consequently, to quote Bath and Duda $\left({ }^{20}\right)$, it is very likely that "the essential difference between large and small shocks is not to be found in the strain release but in the volume within which the release takes place at the same time".

Then, if strain is practically constant, it cannot be the kinematic rheological quantity associated with each shock. In order to overcome this difficulty, Bath and Duda $\left({ }^{20}\right)$ define "deformation" as

$$
D=\bar{\varepsilon} V
$$

where $\bar{\varepsilon}$ is a constant. Referring to aftershock sequences, the deformation of the $i$-th shock is $D_{i}=\bar{\varepsilon} V_{i}$.

Although individual aftershocks occur in different volumes $V_{t}$ within the total aftershock volume $V_{o}$, it makes sense to add their effects when the behavior of $V_{o}$ as a whole is being considered. Deformation, on the other hand, has the dimensions of volume and cannot be used as an equivalent of a kinematic rheological quantity which is dimensionless. Since strain at any point can be expressed by 3 extensions in 3 mutually perpendicolar directions, it can be regarded as deformation per unit volume. Then, an "overall strain" may be defined with reference to the whole volume $V_{o}$ as

$$
\bar{\varepsilon}_{v}=\frac{D}{V_{o}}=\frac{\bar{\varepsilon}}{V_{o}} V
$$

For a given aftershock sequence, since $V_{0}$ is constant, one has for the $i$-th shock.

$$
\bar{\varepsilon}_{v i}=c V_{i} \text {. }
$$

Now, it follows from [9] and [6] that the volume affected by a shock is proportional to the energy released. Therefore, when considering the rock volume $V_{0}$ as a whole, the overall strain is proportional to energy. This is not characteristic of an elastic medium, but of a 
medium whose rheological behavior is plastic. Thus, the overall rheological behavior of the material in the earthquake volume (total aftershock volume) is best described in terms of plasticity. This conclusion is also supported by the experimental results; non-linear (logarithmic) plastic creep under constant load is typically observed in polycrystalline aggregates such as metals and rocks. Tlis plastic creep in a heterogeneous medium causes local stress concentrations that may lead to fracture $\left({ }^{22}\right)$. Fractures during continuous creep have indeed been detected in the laboratory both in metals and in rocks. The experimental results obtained by Lomnitz $\left({ }^{23}\right)$ indicate that the same type of creep occurs also during recovery when the load is removed.

Taking everything together, the following picture of the aftershock process emerges from the preceding considerations.

Under an applied tectonic force, the material in the volume $V_{0}$ is deformed continuously (some foreshocks may occur at points of stress concentration) until the strength limit is reached and a sudden elastic stress release occurs (main shock). This stress drop can be caused either by the formation of a new fracture or by a stick-slip episode along a preexisting fracture. The main shock induces a redistribution of stresses inside the medium, to which the material in the vicinity adjusts in an overall fashion by logarithmic creep. The mechanism of this creep is discontinuous and the process is observed as an aftershock sequence.

\section{REFERENCES}

(1) Scineidegger A. E., Rheology of the Earth, The Basic Problem. of Cieodynamics "Canad. J. Phys. ", 35, 383-397 (1957).

(2) Oyori F., On the Aftershocks of Earthquakes "J. Coll. Sci. Imp. Univ. Tokio ", 7, $111-200$ (1894).

(3) Jомnтт C., Magnitude Stability in Earthquake Sequences, "Bull. Seism. Soc. Am, ", 56, 247-249 (1966).

(4) Gutenberg B. and Richter C. F., Seismicity of the Earth and Associated Phenomena, 2nd. ed. "Princeton Univ. Press." Princeton 1954.

(5) Ranalli G., A Statistical Study of Aftershock Sequences, in press.

(') Benioff H., Earthquakes and Rock Creep, I. "Bull. Seism. Soc. Am.", 41, 31-62 (1951).

(7) Gutenberg B., Physics of the Earth's Interior "Academic Press ", New York 1959.

(8) Pshennikov K. V., Aftershock Mechanism and the Inelastic Properties of the Earth's Crust, Nauka, Moscow (1965) [Engl. Transl. TT 66-32280]. 
( ${ }^{\theta}$ Kuznetsova K. I., and Popov V. V., The Treatment of the Mechanical Properties of the Earth's Crust in a Book by K. V. Pshennikov, "Izv. Phys. Solid. Earth" 9, 618-621 (1967).

${ }^{(10)}$ Mogr K., On the Time Distribution of Aftershocks Accompanying the Recent Hajor Earthquakes in and Near Japan, "Bull. Earthq. Res. Inst. ", 40, 107.124, (1962).

(11) MoGI K., Study of Elastic Shocks Caused by the Fracture of Heterogeneous Materials and its Relations to Earthquake Phenomena, "Bull. Earthq. Res. Inst. ", 40, 125-173 (1962).

${ }^{(12)}$ Mogi K., The Fracture of a Semi-infinite Body Caused by an Inner Stress Origin and its Relation to Earthquake Phenomena, I, "Bull. Earthq. Res. Inst. ", 40, 815-829, (1962).

${ }^{\left({ }^{13}\right)}$ Mogi K., The Fracture of a Semi-infinite Body Caused by an Inner Stress Origin and its Relation to Earthquake Phenomena, II, "Bull. Earthq. Res. Inst. ", 41, 595-614, (1963).

(14) MogI K., Earthquakes and Fractures, "Tectonophysics", 5, 35-55 (1967)

(15) Scholz C. H., Microfracturing and the Inelastic Deformation of Rock in Compression, "J. Geophys. Res. ", 73,1417-1432, (1968).

$\left({ }^{18}\right)$ Scholz C. H., Mechanism of Creep in Brittle Rock, "J. Geophys. Res.", 73, 3295-3302, (1968).

(17) Sсноцz C. H., The Frequency-Magnitude Relation of Microfracturing in Rock and its Relation to Earthquakes, "Bull. Seism. Soc. Am.", 58, 399-415, (1968).

(18) JefFreys Sir H., Aftershocks and Periodicity in Earthquakes, "Gerl. Beitr. Geophys. ", 53, $111-139$, (1938).

(19) Scholz C. H., Microfractures, Aftershocks, and Seismicity, "Bull. Seism. Soc. Am. ", 58, 1117.1130 , (1968).

${ }^{(20)}$ B \&ти M. and DUdA S. J., Earthquake Volume, Fault Plane Area, Seismic Energy, Strain, Deformation and Related Quantities, "Ann. Geofisica ", 17, 353-368, (1964).

$\left({ }^{21}\right)$ Tsuвог C., Earthquake Energy, Earthquake Volume, Aftershock Area, and Strength of the Earth's Crust, "J. Phys. Earth", 4, 63-66, (1956).

(22) Savage J. C., Moiranty B. B., Does Creep Cause Fracture in Britle Rocks?, "J. Geophys. Res. ", 74, 4329-4332, (1969).

${ }^{(23)}$ Lomnitz C., Creep Measurements in Igneous Rock, "J. Geology ", 64, $473-479$, (1956). 